\title{
LONG-TERM RETURNS IN STOCHASTIC INTEREST RATE MODELS: DIFFERENT CONVERGENCE RESULTS.
}

Griselda Deelstra

Vrije Universiteit Brussel

Department of Mathematics

Pleinlaan 2

B-1050 Brussels - Belgium

e-mail: gdeelstr@dwis1.vub.ac.be
Fred Delbaen

ETH Zentrum,

Department of Mathematics

$\mathrm{CH}$ - 8092 Zürich

Switzerland

e-mail: delbaen@math.ethz.ch

Abstract: In this paper, we focus on different convergence results of the long-term return $\frac{1}{t} \int_{0}^{t} r_{u} d u$, where the short interest rate $r$ follows an extension of the Cox, Ingersoll and Ross ${ }^{1}$ model. Using the theory of Bessel processes, we proved the convergence almost everywhere of $\frac{1}{t} \int_{0}^{t} X_{u} d u$, where $\left(X_{u}\right)_{u \geq 0}$ denotes a generalisation of a Besselsquare process with drift. We also studied the convergence in law of the long-term return in order to make some approximations. We observed the convergence in law of the sequence of processes $\left(Y^{n}\right)_{n \geq 1}$ with $\left(Y_{t}^{n}\right)_{t \geq 0}=\left(\sqrt{\frac{-2 \beta^{3}}{\bar{\delta} n}} \int_{0}^{n t}\left(X_{u}+\frac{\delta_{u}}{2 \beta}\right) d u\right)_{t \geq 0}$. By Aldous' criterion, this sequence converges in law to a Brownian motion. These convergence results have some immediate applications.

Keywords: Long-term return, stochastic interest rate, generalized Besselsquare processes, convergence almost everywhere, convergence in law, bond prices.

\section{Introduction}

In this paper, we concentrate on different convergence results of the long-term return $\frac{1}{t} \int_{0}^{t} r_{u} d u$, where the short interest rate $r$ follows an extension of the Cox, Ingersoll and Ross ${ }^{1}$ model. Since it is reasonable to conjecture that the market will influence the reversion level and the volatility coefficient, we assume the reversion level to be stochastic and also take the volatility more general. 
Using the theory of Bessel processes and more specifically results of Pitman-Yor ${ }^{2}$, we proved in Deelstra-Delbaen ${ }^{3}$ the convergence almost everywhere of the longterm return $\frac{1}{t} \int_{0}^{t} X_{u} d u$, where $\left(X_{u}\right)_{u \geq 0}$ denotes the process defined by the stochastic differential equation $d X_{s}=\left(2 \beta X_{s}+\delta_{s}\right) d s+g\left(X_{s}\right) d B_{s}$ with $\beta<0$, with $g$ a positive function, vanishing at zero and satisfying a Hölder condition of order $1 / 2$, and with $\left(\delta_{s}\right)_{s \geq 0}$ a stochastic process satisfying some hypotheses. In case of the generalized Besselsquare processes $(g(x)=2 \sqrt{x})$ with drift parameter $\beta<0$ and stochastic reversion level $\left(\delta_{s}\right)_{s \geq 0}$, we found in Deelstra-Delbaen 4 that - under the right assumptions - the sequence of processes $\left(Y^{n}\right)_{n \geq 1}$ with

$$
\left(Y_{t}^{n}\right)_{t \geq 0}=\left(\sqrt{\frac{-2 \beta^{3}}{\bar{\delta} n}} \int_{0}^{n t}\left(X_{u}+\frac{\delta_{u}}{2 \beta}\right) d u\right)_{t \geq 0}
$$

converges in law to a Brownian motion for $n$ going to infinity. Both theorems have some straightforward applications.

In section 2 , we introduce the family of processes $\left(X_{u}\right)_{u \geq 0}$. The convergence a.e. result is stated in section 3. An immediate application of this theorem is proposed. In the last section, we look at the convergence in law result and we discuss some approximations of the long-term return and of bond prices (see also Deelstra ${ }^{5}$ ). These approximations have applications in finance and in assurance.

In this paper, we omit the long and technical proofs in order to obtain a clear summary of the different convergence results of the long-term return.

\section{Stochastic interest rate models}

We analyse the convergence of the long-term return, using an extension of the Cox, Ingersoll and Ross ${ }^{1}$ stochastic model of the short interest rate $r$. Cox, Ingersoll and Ross express the short interest rate dynamics as

$$
d r_{t}=\kappa\left(\gamma-r_{t}\right) d t+\sigma \sqrt{r_{t}} d B_{t}
$$

with $\left(B_{t}\right)_{t \geq 0}$ a Brownian motion and $\kappa, \gamma$ and $\sigma$ positive constants. It is a wellknown fact that this model has some empirically relevant properties. In this model, $r$ never becomes negative and for $2 \kappa \gamma \geq \sigma^{2}, r$ does not reach zero. For $\kappa>0$ and $\gamma \geq 0$, the randomly moving interest rate is elastically pulled towards the long-term constant value $\gamma$. The volatility increases with the instantaneous interest rate. There is a steady state distribution.

In a mathematical respect, the CIR square root process $r$ is interesting since it is a transformation of a Besselsquare process with drift. Indeed, if we define $X$ by the 
transformation $X=\frac{4}{\sigma^{2}} r$, then $X$ is a Besselsquare process with drift parameter $-\kappa / 2$ and dimension $\frac{4 \kappa \gamma}{\sigma^{2}}$. The many results known about these processes (see e.g. Pitman-Yor ${ }^{2}$ and Revuz-Yor6), convinced us that these processes are very tractable.

However, it is reasonable to conjecture that the market will constantly change the reversion level $\gamma$ and the volatility $\sigma$. A lot of authors already have extended the CIR model in order to reflect the time-dependence caused by the cyclical nature of the economy or by expectations concerning the future impacts of monetary policies. For example, Hull and White ${ }^{7}$ assumed time-dependent parameters; Brennan and Schwartz ${ }^{8}$, Schaefer and Schwartz ${ }^{9}$ and Longstaff and Schwartz 10 suggested twofactor models with a fixed correlation. We take the volatility more general and we allow the reversion level to be stochastic and to have an arbitrary correlation with the short-term interest rate process. In this way, we treat two-factor models where the two factors may have a random correlation.

We consider a family of stochastic processes $X$, which contains the Besselsquare processes with drift, namely $X$ satisfying the stochastic differential equation $d X_{s}=\left(2 \beta X_{s}+\delta_{s}\right) d s+g\left(X_{s}\right) d B_{s}$ with $\delta$ a non-negative measurable and adapted stochastic process, with $\beta<0$ and $g$ a positive function, vanishing at zero and such that there is a constant $b$ such that $|g(x)-g(y)| \leq b \sqrt{|x-y|}$. This stochastic differential equation has a unique non-negative strong solution as soon as $\int_{0}^{t} \delta_{u} d u<\infty$ for all $t \in \mathfrak{R}^{+}$.

\section{Convergence a.e. of the long-term return}

Using the theory of Bessel processes, we found the following theorem, which is very useful for deducing the convergence almost everywhere of the long-term return in quite general situations:

\section{Theorem 1}

Suppose that a probability space $\left(\Omega,\left(\boldsymbol{F}_{\mathrm{t}}\right)_{t \geq 0}, \boldsymbol{P}\right)$ is given and that a Brownian motion $\left(B_{t}\right)_{t \geq 0}$ is defined on it. A stochastic process $X: \Omega \times \mathfrak{R}^{+} \rightarrow \mathfrak{R}^{+}$is assumed to satisfy the stochastic differential equation

$$
d X_{s}=\left(2 \beta X_{s}+\delta_{s}\right) d s+g\left(X_{s}\right) d B_{s}
$$

with $\beta<0$ and $g: \Re \rightarrow \Re^{+}$a function, vanishing at zero and such that there is a constant $b$ with $|g(x)-g(y)| \leq b \sqrt{|x-y|}$. 
The measurable and adapted process $\delta: \Omega \times \mathfrak{R}^{+} \rightarrow \mathfrak{R}^{+}$is assumed to satisfy:

$$
\frac{1}{s} \int_{0}^{s} \delta_{u} d u \stackrel{\text { a.e. }}{\longrightarrow} \bar{\delta} \text { with } \bar{\delta}: \Omega \rightarrow \mathfrak{R}^{+} .
$$

Under these conditions, the following convergence almost everywhere holds:

$$
\frac{1}{s} \int_{0}^{s} X_{u} d u \stackrel{\text { a.e. }}{\longrightarrow} \frac{-\bar{\delta}}{2 \beta} \text {. }
$$

The proof of the convergence a.e. result relies on the theory of stochastic differential equations and on Kronecker's lemma. The interested reader is referred to "Long-term returns in stochastic interest rate models" 3 .

We immediately turn to an application of theorem 1 . We consider the long-term return in the following CIR two-factormodel:

$$
\begin{aligned}
& d r_{t}=\kappa\left(\gamma_{t}-r_{t}\right) d t+\sigma \sqrt{r_{t}} d B_{t} \\
& d \gamma_{t}=\tilde{\kappa}\left(\tilde{\gamma}-\gamma_{t}\right) d t+\tilde{\sigma} \sqrt{\gamma_{t}} d \tilde{B}_{t}
\end{aligned}
$$

with $\left(B_{t}\right)_{t \geq 0}$ and $\left(\tilde{B}_{t}\right)_{t \geq 0}$ two Brownian motions and with $\kappa, \tilde{\kappa}, \sigma, \tilde{\sigma}$ and $\tilde{\gamma}$ positive constants. The short interest rate process has a reversion level which is a stochastic process itself. Since the reversion level $\left(\gamma_{t}\right)_{t \geq 0}$ follows a CIR square root process, we know that the stochastic reversion level $\left(\gamma_{t}\right)_{t \geq 0}$ is elastically pulled towards the longterm constant value $\tilde{\gamma}$. We are interested in the convergence of the long-term return $\frac{1}{t} \int_{0}^{t} r_{u} d u$.

We remark that we do not need any assumptions about the correlation between the Brownian motions of the instantaneous interest rate and of the stochastic reversion level process. They even may have an arbitrary random correlation. We stress this fact because it is not trivial. Most authors of two-factormodels require, for technical reasons, that the Wiener processes are uncorrelated or have a deterministic and fixed correlation.

It is clear that $\left(\gamma_{t}\right)_{t \geq 0}$ satisfies the conditions of theorem 1 and that consequently

$$
\frac{1}{s} \int_{0}^{s} \gamma_{u} d u \stackrel{\text { a.e. }}{\longrightarrow} \tilde{\gamma} \text {. }
$$

We now consider the instantaneous interest rate $r$ itself and verify whether the assumptions of theorem 1 are fulfilled by $r$. Trivially, the volatility function $g(x)=\sigma \sqrt{x}$ satisfies a Hölder condition of order $1 / 2$ and $\beta=-\kappa / 2$ is strictly negative. The process $\left(\kappa \gamma_{t}\right)_{t \geq 0}$ is measurable and adapted and clearly,

$$
\frac{1}{s} \int_{0}^{s} \kappa \gamma_{u} d u \stackrel{\text { a.e. }}{\longrightarrow} \kappa \tilde{\gamma} \text {. }
$$


Therefore, we may invoke theorem 1 to obtain

$$
\frac{1}{s} \int_{0}^{s} r_{u} d u \stackrel{a e .}{\longrightarrow} \tilde{\gamma}
$$

We conclude that the long-term return converges a.e. to $\tilde{\gamma}$, the long-term constant value towards which the reversion level process $\left(\gamma_{t}\right)_{t \geq 0}$ is pulled in this twofactormodel.

\section{Convergence in law}

Encouraged by the convergence almost everywhere result, we analysed the convergence in law in "Long-term returns in stochastic interest rate models: Convergence in law" 4 . We are interested in the convergence in law since it is useful to know how the long-term return is distributed in the limit so that one can find approximations.

We suppose the same hypotheses as in theorem 1, so that we can apply the previous convergence theorem. Adding some assumptions about the second moment of the stochastic reversion level process $\delta$, we find the following theorem:

Theorem 2

Suppose that a probability space $\left(\Omega,\left(\boldsymbol{F}_{\mathrm{t}}\right)_{t \geq 0}, \boldsymbol{P}\right)$ is given and that a stochastic process $X: \Omega \times \mathfrak{R}^{+} \rightarrow \mathfrak{R}^{+}$is defined by the stochastic differential equation

$$
d X_{s}=\left(2 \beta X_{s}+\delta_{s}\right) d s+2 \sqrt{X_{s}} d B_{s}
$$

with $\left(B_{t}\right)_{t \geq 0}$ a Brownian motion and $\beta<0$. Let us make the following assumptions

about the adapted and measurable process $\delta: \Omega \times \mathfrak{R}^{+} \rightarrow \mathfrak{R}^{+}$:

* $\frac{1}{s} \int_{0}^{s} \delta_{u} d u \stackrel{\text { a.e. }}{\longrightarrow} \bar{\delta}$ where $\quad \bar{\delta}$ is a strictly positive real number;

* $\sup _{t \geq 1} \frac{1}{t} \int_{0}^{t} \mathbf{E}\left[\delta_{u}^{2}\right] d u \leq k$ with $k$ a constant independent of $t$;

* For all $a \in \mathfrak{R}^{+} \lim _{t \rightarrow \infty} \frac{1}{t} \int_{t-a}^{t} \mathbf{E}\left[\delta_{u}^{2}\right] d u=0$.

Under these conditions, the following convergence in distribution holds:

$$
\left(\sqrt{\frac{-2 \beta^{3}}{\bar{\delta} n}} \int_{0}^{n t}\left(X_{u}+\frac{\delta_{u}}{2 \beta}\right) d u\right)_{t \geq 0} \stackrel{L}{\longrightarrow}\left(B_{t}^{\prime}\right)_{t \geq 0}
$$

with $\left(B_{t}^{\prime}\right)_{t \geq 0}$ a Brownian motion.

Remark that there is no assumption about the correlation between the process $X$ and the reversion level process $\delta$. 
In order to prove the convergence in law, we have checked that the sequence $\left(Y^{n}\right)_{n \geq 1}$, defined by $Y_{t}^{n}=\sqrt{\frac{-2 \beta^{3}}{\bar{\delta} n}} \int_{0}^{n t}\left(X_{u}+\frac{\delta_{u}}{2 \beta}\right) d u$, converges to a Brownian motion $\left(B_{t}^{\prime}\right)_{t \geq 0}$ in the sense of finite distributions. Afterwards, we have shown that the sequence is weakly relatively compact by using Aldous' criterion for tightness.

We now concentrate on some applications of the convergence in law. We consider the possibility of approximating $\int_{0}^{t} r_{u} d u$ since this integral appears in bond prices, accumulation and discounting factors, etcetera.

As an example, let us retake the generalized Cox-Ingersoll-Ross two-factor model, which is introduced in the previous section. Since we are interested in the convergence of the long-term return $\frac{1}{t} \int_{0}^{t} r_{u} d u$, we verify whether the assumptions of theorem 2 are fulfilled.

Defining $X$ by the transformation $X=\frac{4}{\sigma^{2}} r$, we find that $X$ satisfies the stochastic differential equation

$$
d X_{u}=\left(\frac{4 \kappa \gamma_{u}}{\sigma^{2}}+2\left(-\frac{\kappa}{2}\right) X_{u}\right) d u+2 \sqrt{X_{u}} d B_{u} .
$$

Since we know that the hypotheses of theorem 1 are fulfilled, it only remains to check the conditions about the second moment of $\left(\delta_{t}\right)_{t \geq 0}=\left(\frac{4 \kappa \gamma_{t}}{\sigma^{2}}\right)_{t \geq 0}$. Since $\left(\gamma_{t}\right)_{t \geq 0}$ follows a CIR square root process, its second moment is given by:

$$
\mathbf{E}_{\gamma_{0}}\left[\gamma_{s}^{2}\right]=\left(2 \tilde{\kappa} \tilde{\gamma}+\tilde{\sigma}^{2}\right)\left[\frac{\gamma_{0}-\tilde{\gamma}}{\tilde{\kappa}} \mathrm{e}^{-\tilde{\kappa} s}+\frac{\tilde{\gamma}}{2 \tilde{\kappa}}\right]+\mathrm{e}^{-2 \tilde{\kappa} s}\left[\gamma_{0}^{2}+\frac{\tilde{\gamma}-\gamma_{0}^{2}}{2 \tilde{\kappa}}\left(2 \tilde{\kappa} \tilde{\gamma}+\tilde{\sigma}^{2}\right)\right]
$$

and a technical calculation leads to the results that for all $a \in \mathfrak{R}^{+}$

$$
\lim _{t \rightarrow \infty} \frac{1}{t} \int_{t-a}^{t} \mathbf{E}\left[\delta_{u}^{2}\right] d u=0
$$

and that

$$
\sup _{t \geq 1} \frac{1}{t} \int_{0}^{t} \mathbf{E}\left[\delta_{u}^{2}\right] d u \leq k
$$

with $k$ a constant independent of $t$.

Consequently, we may apply theorem 2 and we find that

$$
\left(\frac{\kappa}{\sigma \sqrt{\tilde{\gamma} n}} \int_{0}^{n t}\left(r_{u}-\gamma_{u}\right) d u\right)_{t \geq 0} \stackrel{L}{\longrightarrow}\left(B_{t}^{\prime}\right)_{t \geq 0} .
$$


This is not a trivial result since $r$ and $\gamma$ may have an arbitrary, random correlation.

Given this convergence result, we consider the possibility of approximating $\int_{0}^{t} r_{u} d u$ or, more general, of approximating $\int_{0}^{t} X_{u} d u$ where $X$ is defined as in theorem 2. The convergence in law result inspired us to approximate

$$
\sqrt{\frac{-2 \beta^{3}}{\delta n}} \int_{0}^{n t}\left(X_{u}+\frac{\delta_{u}}{2 \beta}\right) d u
$$

by $B_{t}^{\prime}$ for $n$ large enough.

Using the scaling property of Brownian motion, namely $\sqrt{n} B_{t / n}^{\prime} \stackrel{d}{=} B_{t}^{\prime}$, we estimate

$$
\int_{0}^{t} X_{u} d u \text { by }-\int_{0}^{t} \frac{\delta_{u}}{2 \beta} d u+\sqrt{\frac{\bar{\delta}}{-2 \beta^{3}}} B_{t}^{\prime} \text {. }
$$

As $\frac{1}{t} \int_{0}^{t} \delta_{u} d u \stackrel{\text { a.e. }}{\longrightarrow} \bar{\delta}$, we obtain for $t$ large enough that

$$
\int_{0}^{t} X_{u} d u \approx-\frac{\bar{\delta}}{2 \beta} t+\sqrt{\frac{\bar{\delta}}{-2 \beta^{3}}} B_{t}^{\prime} .
$$

Therefore, we approximate $\int_{0}^{t} X_{u} d u$ by the constant convergence a.e. limit of the long-term return times $t$ plus a scaled Brownian motion.

A drawback of this estimator is that the moments of $\int_{0}^{t} X_{u} d u$ do not equal those of the estimator, although they are the same asymptotically. If the period observed is large enough, this is satisfying. If the objective is to approximate the distribution of the long-term return of an investment made at time 0 , it seems appropriate to approximate $\int_{0}^{t} X_{u} d u$ by a scaled Brownian motion with drift since the Central Limit Theorems are applicable on long-term.

However, one of our objectives is to find approximations of bond prices for all maturities. Therefore, the moments of $\int_{0}^{t} X_{u} d u$ and of the estimator should be equal for all $t \in \mathfrak{R}^{+}$. If one looks at a zero-coupon bond price in a risk-neutral setting, namely

$$
\mathbf{E}_{X_{0}}\left[\exp \left(-\int_{0}^{t} X_{u} d u\right)\right] \exp \left(\frac{\bar{\delta}}{2 \beta} t-\frac{\bar{\delta}}{4 \beta^{3}} t\right)
$$

a second drawback of the approximation immediately appears. It is not realistic that an estimation of a bond price is independent of the current short interest rate $X_{n}$. 
In case of the Cox-Ingersoll-Ross one-factor model, the approximating bond price equals:

$$
\mathbf{E}_{r_{0}}\left[\exp \left(-\int_{0}^{t} r_{u} d u\right)\right] \exp \left(\gamma t\left(\frac{\sigma^{2}}{2 \kappa^{2}}-1\right)\right)
$$

This approximation is a decreasing function of the speed of adjustment parameter $\kappa$, where in the case of the Cox-Ingersoll-Ross model, two cases are distinguished: for $r_{0}<\gamma$, the bond price is a decreasing function of the parameter $\kappa$, and for $r_{0}>\gamma$, it is an increasing function of $\kappa$. In Deelstra-Delbaen ${ }^{4}$, we showed by comparing these approximating bond prices with the exact bond prices (see Pitman-Yor ${ }^{2}$ or Cox, Ingersoll and Ross ${ }^{1}$ ), that there is an underestimation of the bond prices if $r_{0}<\gamma$ and an overestimation if $r_{0}>\gamma$.

Therefore, we searched for an improved approximation. In "Long-term returns in stochastic interest rate models: Applications" 5 , we propose the approximation

$$
\int_{0}^{t} X_{u} d u \approx \int_{0}^{\mathrm{t}} \mathbf{E}\left[X_{u}\right] d u+\sqrt{\frac{\bar{\delta}}{-2 \beta^{3}}} B_{t}^{\prime} .
$$

In this case, the expectation value is equal for all $t$ and the variance still is asymptotically equal.

The improved approximation has some interesting applications in insurance. For example, the expected values, the variances and the skewness of various insurance products can be approximated in quite general stochastic interest rate models. Following the method of Parker 11,12, $n$-year temporary life assurances, whole-life assurances and endowment assurances have been treated in Deelstra 5 in case of a generalisation of the Cox-Ingersoll-Ross model.

\section{References}

1. J.C. Cox, J.E. Ingersoll and S.A. Ross, 'A theory of the term structure of interest rates', Econometrica, 53, 385-407 (1985).

2. J. Pitman and M. Yor, 'A decomposition of Bessel Bridges', Zeitschrift für Wahrscheinlichkeitstheorie und Verwandte Gebiete, 59, 425-457 (1982).

3. G. Deelstra and F. Delbaen, 'Long-term returns in stochastic interest rate models', Insurance: Mathematics and Economics, 17, 163-169 (1995).

4. G. Deelstra and F. Delbaen, 'Long-term returns in stochastic interest rate models: Convergence in law', Stochastics and Stochastics Reports, 55, 253-277 (1995).

5. G. Deelstra, 'Long-term returns in stochastic interest rate models: Applications', in Proceedings of the 5th AFIR International Colloquium, Brussels, $709-730$ (1995). 
6. D. Revuz and M. Yor, Continuous Martingales and Brownian Motion, SpringerVerlag, Berlin-Heidelberg, New-York, 1991.

7. J. Hull and A. White, 'Pricing Interest-Rate-Derivative Securities', The Review of Financial Studies, 3 (4), 573-592 (1990).

8. M.J. Brennan and E.S. Schwartz, 'An Equilibrium Model of Bond Pricing and a Test of Market Efficiency', Journal of Financial and Quantitative Analysis, 17, 301329 (1982).

9. S.M. Schaefer and E.S. Schwartz, 'A two-factor model of the term structure: An approximate analytical solution', Journal of Financial and Quantitive Analysis, 19, 413-424 (1984).

10. F.A. Longstaff and E.S. Schwartz, 'Interest rate volatility and the term structure: a two-factor general equilibrium model', The Journal of Finance, 47 (4), 1259-1282 (1992).

11. G. Parker, 'Distribution of the present value of future cash flows', in Proceedings of the 3rd AFIR International Colloquium, Rome, 831-843 (1992).

12. G. Parker, 'Moments of the present value of a portfolio of policies', Scandinavian Actuarial Journal, 53-67 (1994).

13. G. Deelstra, 'Long-term returns in stochastic interest rate models', Ph.D. thesis, Vrije Universiteit Brussels (1995). 